\title{
PHARMACOTHERAPEUTIC MANAGEMENT OF CHRONIC VIRAL HEPATITIS
}

\author{
Gabriela Mitea $^{1 *}$, Marius Daniel Radu ${ }^{2}$, Ana Maria Ionescu ${ }^{3}$, Nicoleta Blebea ${ }^{1}$ \\ ${ }^{1}$ Ovidius University of Constanta, Faculty of Pharmacy, Constanta, Romania \\ ${ }^{2}$ Ovidius University of Constanta, Faculty of Natural and Agricultural Sciences, Constanta, Romania \\ ${ }^{3}$ Ovidius University of Constanta, Faculty of Medicine, Constanta, Romania \\ *Corresponding author: Gabriela Mitea, gegiugabriela@yahoo.com
}

\begin{abstract}
In infectious diseases, viral hepatitis has an increased incidence, being one of the leading causes of death worldwide, being a real indicator of the socio-economic and hygieniccultural standard of a geographical area. The World Health Organization (WHO) admits the following types of hepatitis viruses: A, B, B + D, C, E, F and G. Among the viral entities recognized by the WHO, a special importance in terms of incidence, evolution over time with the risk of chronicity and the therapeutic options are presented by hepatitis B and C. There is currently an effective vaccine as well as treatment for hepatitis B. There is no vaccine for hepatitis $\mathrm{C}$, but lately sustained efforts have been made to treat this disease. Also, the introduction of drugs known as direct-acting antivirals makes it possible to cure over $90 \%$ of patients within 2-3 months. But in many countries, current drug policies, regulations and prices keep treatment out of the reach of most people with hepatitis. Eradication of viral hepatitis is possible if greater emphasis is placed on prevention, diagnosis and treatment. Vaccination is very important, as it is possible for types A and B of hepatitis. Early diagnosis is also extremely important, given that there are currently very effective drugs that can prevent the development of liver cancer. The WHO also noted that about two million people worldwide become ill each year due to the reuse of syringes and stressed the importance of checking blood donors to see if they are not carriers of hepatitis B or C viruses. The purpose of this study is to highlight the pharmacological treatment and the main therapeutic and prevention schemes currently used for patients with these liver diseases. The paper was based on the most current search methods of the latest international information about the treatment of hepatitis (by electronic search using Scirus, Pubmed, Web of Science SciFinder and GoogleScholar). We also consulted the global literature cited in the hepatitis database of the World Health Organization (WHO) updated frequent from the current literature on this topic.
\end{abstract}

Keywords. hepatitic virus, treatment, prophylaxis.

\section{Introduction:}

Due to its regenerative capacity, the liver is the most resistant organ of the human body, but like any other, its damage can lead to certain diseases. Among the most common diseases are viral hepatitis that affects the liver, due to lesions present in the liver cells. The liver helps to eliminate toxins present in the blood, but its inflammation prevents it from functioning properly. Therefore, bilirubin, one of the residual substances, begins to accumulate in the tissues and cells. Bilirubin is actually the substance that stains the epidermis and eyeballs of people with hepatitis yellow. Inflammation of the liver is accompanied by fever, nausea, vomiting, muscle aches. The main forms of hepatitis can be divided into two typologies: acute hepatitis, whose evolution is rapid and chronic hepatitis, which usually occurs after the acute phase. If acute hepatitis does not heal in about 6 months, it becomes chronic $[1,2,3]$. 


\section{Diagnosis}

The discovery of hepatitis is usually causal, as a result of laboratory tests performed to diagnose other diseases. If it is symptomatic, chronic hepatitis is characterized by digestive disorders, loss of appetite, fatigue, joint pain, weight loss. Palpation may increase the volume of the liver. The diagnosis is confirmed by liver biopsy. The determining factors coincide with those that cause acute hepatitis: different types of viruses (B, C, D), excessive alcohol consumption, prolonged administration of drugs or toxic substances [4].

\section{Classification of the most common types of chronic viral hepatitis}

4. Chronic hepatitis is caused by chronic hepatitis $\mathrm{B}$ or $\mathrm{C}$ virus infection, as the hepatitis A virus does not become chronic. In rarer cases it may have an autoimmune origin or may accompany autoimmune diseases. Chronic hepatitis, over time, can cause liver cirrhosis. The severity of the installed inflammatory condition may be one of the factors underlying the onset of the disease [3]. The most common types of viral hepatitis are acute and chronic, which are based on a series of risk factors, such as viral, toxic and autoimmune. Given that hepatitis $\mathrm{C}$ in particular can develop without being accompanied by a particular symptom, it is very easy for it to become chronic [4].

Histopathological classification:

- persistents chronic hepatitis, a consequence of acute hepatitis, often asymptomatic, is usually diagnosed by chance, following blood tests;

- chronic lobular hepatitis, which is characterized by the appearance of necrosis at the periphery of the hepatic lobes;

- chronic active hepatitis, characterized by the progression of necrosis $[2,4]$.

\subsection{Hepatitis $A$}

Hepatitis A is a viral infection that causes inflammation and liver damage. Inflammation is the swelling that occurs when body tissues become injured or infected. Inflammation can affect the organs. Viruses invade normal cells in the body and cause infections that can spread from person to person. The hepatitis A virus usually spreads through contact with food or water that has been contaminated in the stool of an infected person. Hepatitis A is an acute or short-lived infection, which means that symptoms usually improve after a few weeks without treatment, which is why it does not lead to more complicated conditions such as cirrhosis $[5,6]$.

\subsubsection{Hygienic-dietary treatment}

It is based on:

- avoiding prolonged physical exertion and stress;

- bed rest after each meal for at least $1 / 2-1$ hour;

- observance of sleep hours of at least 8 hours;

- consumption of many vegetables and fruits;

- stopping treatment with hepatotoxic drugs if they are not prescribed by a doctor;

- maintaining cholesterol, triglyceride and blood sugar levels within normal limits;

- administration of vitamins and phospholipids and consumption of hepatotrophic medicinal plants, 510 days per month or at the doctor's indication; 
- repetition of liver function tests; blood clotting tests; the rate of viral multiplication and neoplastic markers at 4-6 months or as directed by the doctor;

- repeat the abdominal ultrasound at 4-6 months or at the doctor's indication;

- notifying the medical staff of the infection with the hepatitis B, C, or B and C virus $[5,6,7]$.

\subsubsection{Pharmaceutical treatment}

Being a form of acute hepatitis, the specific medication for hepatitis $\mathrm{A}$ is limited to treating the symptoms and careful monitoring of the liver. Very rarely, severe liver disease may occur as a result of hepatitis A virus infection. Then medication, hospitalization or transplant are needed. Sometimes, if drugs are not used, liver damage can be life-threatening. [8]. There is no specific treatment, but only symptomatic based on the administration of vitamins and liver protectors. Rigorous hygiene can reduce the risk of contamination, but does not provide effective and safe protection. Almost all patients with hepatitis A return to normal and gain immunity to this infection throughout their lives $[8,9]$. The covalescence period is 30-60 days, representing the period in which a periodic, clinical and laboratory control is made and healing is consolidated. The administration of immunoglobulins is a fast, expensive and time-limited method (3-6 months of protection) [6]. Vaccination is the only truly safe, fast and effective method, conferring immunity over a long period of time against hepatitis A [1]. Doctors diagnose hepatitis A based on symptoms and a blood test that detects antibodies to the hepatitis A virus. Treatment includes rest, eating healthy foods, taking medications to relieve symptoms and drinking plenty of fluids [5]. Alcohol consumption should be avoided until complete recovery. Prophylaxis of hepatitis A virus infection can be achieved by vaccination against this disease or by taking other measures that can reduce the chances of infection. If there is a history of hepatitis A, reinfection is excluded but not infection with other types of viral hepatitis $[5,6,9]$.

\subsection{Hepatitis $B$}

Acute hepatitis B is a short-term disease that occurs within the first 6 months of exposure to the hepatitis $B$ virus. Acute infection can range in severity, from minor to severe symptoms that can lead to hospitalization. In some cases, especially in adults, the virus disappears without treatment. Healed people gain immunity for the rest of their lives. Sometimes, acute infection can lead to chronic infection. Chronic hepatitis B is based on long-term infection with the hepatitis B virus. Over time, chronic hepatitis B can lead to liver damage, cirrhosis, liver cancer or even death. Infants and young children are most likely to develop chronic hepatitis B infection. There is currently no cure for this condition, only a vaccine can prevent hepatitis B. It is important to take certain precautions to prevent the spread of the virus $[10,11]$.

\subsubsection{Hygienic-dietary treatment}

- exercise for 10 minutes, several times a day;

- increases the duration and level of progressive physical effort - up to 30 minutes daily, from 3 to 5 days a week;

- it starts with a 10-minute walk or a 15-minute swimming session;

- no sustained physical effort should be made suddenly or until exhaustion;

Sudden weight loss promotes the development of hepatitis B virus, as well as obesity, therefore, a balanced diet is most indicated [11]. 
- consumption of fresh fruits is recommended, due to the vitamins and antioxidants they contain, because canned fruits usually have a large amount of sugar;

- consumption of fresh vegetables, in the form of salads;

- alcohol consumption, even in small quantities or occasionally is strictly forbidden to patients with hepatitis B;

- excessive alcohol consumption for long and constant periods of time can lead to liver cirrhosis and even liver cancer. The risk is higher for those who already have hepatitis B.who have a weakened body and damaged liver $[10,12,13]$.

\subsubsection{Pharmaceutical treatment}

Acute hepatitis B infection usually does not require treatment. However, in rare cases, the infection can cause life-threatening liver failure. Patients with hepatic impairment due to acute hepatitis B should be evaluated for liver transplantation [10].

If a person is chronically infected with hepatitis B and has few signs or symptoms of complications, medications are not usually used. These patients are closely monitored and have regular blood tests. One of the tests measures the amount of viral deoxyribonucleic acid (DNA) in the blood [11]. Doctors will recommend treatment if there are signs that the virus is starting to cause damage or if the viral load is high. Another reason to prescribe medication is if the patient has a positive test for hepatitis B antigen $(\mathrm{HBeAg})$ in the blood. $\mathrm{HBeAg}$ is associated with an increased risk of liver disease progression and its complications. In the case of chronic hepatitis B, the role of treatment is to reduce the risk of complications, such as liver failure and cirrhosis. Specific drugs for the treatment of chronic hepatitis B are based on interferons and nucleoside/nucleotide analogues [12,13].

The drug therapy that is administered depends on the clinical form: Lamivudine $100 \mathrm{mg} /$ day, alone or in combination with Interferon alfa $2 \mathrm{a}$ or $2 \mathrm{~b}$ (Intron A 2.5-5.000.000 DI 3 times / week, for 4-6 Roferon A 4.500.000 DI / week for 6 months or pegylated Interferon $180 \mathrm{~g} /$ week) [13]. The duration of therapy is at least 3 months, up to several years, depending on the clinical and biological evolution - GPT remission, inflammatory process, circulating DNA titer. Ideally, it should be initiated as early as possible (in the first 4-6 months) before viral DNA integration into the cell genome occurs. Throughout antiviral therapy, regular monitoring of the patient is required to detect adverse effects (hematological more frequently) and to evaluate the therapeutic response - clinical and laboratory controls every 2 months [12].

\subsection{Hepatitis $C$}

Hepatitis $\mathrm{C}$ is defined as a liver infection caused by the hepatitis $\mathrm{C}$ virus. It ranges from mild symptoms that last for several weeks to severe illness for life [14]. Hepatitis $C$ is not accompanied by certain signs and symptoms, even the health of the organs begins to be affected. The levels of liver enzymes (those that measure inflammation of the liver) of a person infected with the hepatitis $\mathrm{C}$ virus can be slightly elevated or normal. In some countries, such as the United States, hepatitis C virus infection is the most common reason for liver transplantation. A significant number of people with chronic hepatitis $\mathrm{C}$ can develop cirrhosis or liver cancer. [15].

\subsubsection{Hygienic-dietary treatment}

- foods rich in iron (chicken liver and organs) should also be avoided. This is because patients suffering from chronic hepatitis $\mathrm{C}$ have problems in the process of removing iron from the body. Therefore, excess iron may occur, which is not at all beneficial for the blood and organs $[16,17,18]$;

- avoiding food poisoning, which can lead to liver failure;

- the diet should contain carbohydrates with low glycemic index, as few refined sugars as possible, rather fresh fruits, but in small quantities and not very sweet; 
- recommendation of consumption of whole grains, lean meat proteins, unfermented skimmed dairy products;

- fast food, too salty, rich in trans fats or hydrogenated is forbidden [19,20];

- a high-fat diet promotes the onset of hepatic steatosis, also known as fatty liver disease. Fatty liver, in combination with the hepatitis $\mathrm{C}$ virus, can accelerate the degradation of liver function, causing cirrhosis;

- avoiding the accumulation of extra kilograms, because this is how the liver is loaded with fat $[14,16]$; If interferon-based or antiviral-based treatment is given, the patient may experience a loss of appetite or side effects such as nausea $[19,20]$.

Patients diagnosed with hepatitis $\mathrm{C}$ should avoid some dietary supplements as they have a potentially harmful effect on the liver as supplements based on colloidal silver and green tea extract [20].

Vitamin D deficiency is frequently associated with liver disease and has even been correlated with the severity of liver disease. The metabolic, anti-inflammatory and anti-fibrotic properties of vitamin D provide plausible mechanisms by which this can have an impact on the various stages of progression and severity of the disease. in view of the status of vitamin $\mathrm{D}$, its severe deficiency requires vitamin $\mathrm{D}$ supplementation for the specific treatment of this condition $[17,20]$.

- avoiding the administration of vitamin B12;

- coffee consumption can be useful in reducing fibrosis and oxidative DNA damage and stabilizing chromosomes $[14,15]$.

\subsubsection{Pharmaceutical treatment}

Hepatitis $\mathrm{C}$ can often be successfully treated by following a treatment that involves taking different combinations of medications for several months. The aim is to eliminate the virus from the blood $[15,16]$. Complete therapy lasts a maximum of 1 year, although the recommended duration of treatment depends on the viral genotype. For example, people with genotype 2 or 3 can receive treatment for only 24 weeks. For patients with genotype 1, the most common type (99\%, according to the latest studies), the treatment usually lasts 48 weeks. It may be necessary to perform another blood test after a few months to see if the body is fighting the virus $[14,20]$.

The current treatment for chronic hepatitis $\mathrm{C}$ may include two therapies, although, globally, triple therapy has already been implemented, for which the chances of response are almost double in patients without previous treatment, while for patients with failure to previous therapy, triples the rates of sustained viral response, ie favorable response $[15,16]$. Until recently, the treatment of hepatitis C involved two main drugs: pegylated interferon (a drug that encourages the immune system to attack the virus, but also prevents HCV from multiplying) and ribavirin (an antiviral drug that stops the virus from reproducing) $[14,16]$. Hepatitis $C$ is the leading cause of liver cancer in Europe and can lead to the need for liver transplantation, an extremely serious, costly and life-saving operation. The most important thing is the early discovery of the disease and the start of treatment $[14,16,17]$.

Adults over the age of 40 who have had a blood transfusion should see a doctor and have a simple lifesaving anti-HCV antibody test. Moreover, even in the world, triple therapy has already been overcome [20]. Internationally renowned specialists, said that soon, patients will have access to new therapies, which will cure $100 \%$ hepatitis C $[19,20]$.

\section{Prophylactic treatment}

Viral hepatitis $\mathbf{A}$ and $\mathbf{E}$ can be prevented by:

- observance of personal hygiene (washing hands before eating and after using the toilet);

- consumption of water from safe sources;

- keeping the household clean, washing and disinfecting toys, cleaning and disinfecting wells;

- individual use of personal hygiene items; vaccination against viral hepatitis A (the vaccine is optionally administered according to epidemiological indications) [1,2].

Viral hepatitis B, C, D and $\mathbf{G}$ can be prevented by: 
- immunization against hepatitis B (vaccination is accessible, free and included in the National Immunization Program; children are vaccinated in the first 24 hours, at 2 months, 4 months and 6 months);

- reducing the risk of sexual transmission (through abstinence, fidelity to one partner and protected sex);

- vigilance during manicure, pedicure, tattoo, piercing.

- Improved sanitation, food safety and immunization are the most effective methods to combat hepatitis A $[1,2]$.

The spread of hepatitis A can be reduced by:

- safe drinking water supply;

- correct disposal of wastewater;

- personal hygiene practices, such as regular hand washing with safe water [1,2].

Several hepatitis A vaccines are available internationally. They are all similar in terms of how to protect people from viruses and side effects. No vaccine is licensed for children under 1 year. Almost $100 \%$ of people develop protective levels of antibodies against the virus within 1 month after a single dose of vaccine.

Even after exposure to the virus, a single dose of vaccine, within 2 weeks of contact with the virus, has protective effects $[1,2]$. However, manufacturers recommend two doses of vaccine to provide long-term protection from about 5 to 8 years after vaccination. Millions of people have been immunized worldwide without serious side effects.

Vaccination efforts should be complemented by health education to improve sanitation and food hygiene and safety practices [1,2]. It is recommended that all newborns receive the hepatitis B vaccine as soon as possible after birth, preferably within 24 hours. The birth dose should be followed by 2 or 3 doses. The complete vaccine series induces levels of protective antibodies in more than $95 \%$ of infants, children and young adults.

There is no vaccine for hepatitis $\mathrm{C}$, therefore, the prevention of $\mathrm{HCV}$ infection depends on reducing the risk of exposure to the virus $[1,2]$.

The following WHO-recommended some strategies that can significantly reduce the risk of infection with hepatitis $\mathrm{C}$ virus:

- washing hands and using gloves;

- safe disposal of waste and sharp objects;

- reducing the injection or giving up drug use would eliminate the main route of transmission of the virus;

- testing of donated blood for hepatitis C (such as HIV and syphilis);

- training of medical staff;

- promoting the correct and consistent use of condoms [1,2].

Secondary and tertiary prevention. For people infected with the hepatitis C virus, the WHO recommends:

- education and counseling on care and treatment options;

- immunization with the hepatitis B vaccine to prevent co-infection with these hepatitis viruses;

- adequate medical management, including antiviral treatment, if applicable $[1,2]$.

\section{Conclusions}

Mankind has ignored hepatitis and the danger it poses, says representatives of WHO, who warns that it is time to implement a global response to the problem of hepatitis, at a level similar to that generated by the fight against other communicable diseases, such as HIV/AIDS and tuberculosis.

The number of drugs approved for the treatment of HBV infection has increased in recent years. Due to this multitude of therapeutic options, stopping the progression of HBV infection and preventing prophylaxis can be resolved if the infection is treated effectively and in a timely manner.

From the introduction of interferon monotherapy to the current combination treatment with PEGinterferon and nucleoside or nucleotide analogues, the sustained virological response has significantly increased. However, the many side effects of interferon therapy lead to discontinuation of treatment. 
That is why new solutions are needed to fight HBV infections. The goal for the future is to develop a treatment with fewer side effects and greater antiviral efficacy.

Specialists say that the treatment based on interferon is the only approved for the treatment of chronic $\mathrm{HCV}$ infection. That contains three distinct antiviral mechanisms, which act synergistically to attack the virus in three separate stages of its life cycle.

Treatment has been approved for administration to patients with chronic hepatitis $\mathrm{C}$ virus infection, genotype 1 (GT1), including those with compensated cirrhosis of the liver, HIV-1 co-infected patients, patients undergoing opiate dependence treatment and patients receive a liver transplant, according to a statement.

The theoretical importance of this study is represented by the presentation of new, complementary and alternative drug therapies for the development of strategies for the optimal management and treatment of viral hepatitis. In addition to following a treatment (combination therapy) to fight the virus, lifestyle changes are also needed to prevent continuous liver damage.

\section{References}

[1] G.W. WEBB, S. KELlY, H.R. DALTON, Hepatitis A and Hepatitis E: Clinical and Epidemiological Features, Diagnosis, Treatment, and Prevention. Clin Microbiol Newsl. 2020 Nov 1;42(21):171-179. doi: 10.1016/j.clinmicnews.2020.10.001. Epub 2020 Oct 22. PMID: 33110280; PMCID: PMC7581387;

[2] D. CASTANEDA, A.J. GONZALEZ, M. ALOMARI, K. TANDON, X.B. ZERVOS, From hepatitis A to E: A critical review of viral hepatitis. World J Gastroenterol. 2021 Apr 28;27(16):1691 1715. doi: 10.3748/wjg.v27.i16.1691. PMID: 33967551; PMCID: PMC8072198;

[3] D. B. ALASTAIR, FERRELL L. D., HÜBSCHER S. G., MacSween's Pathology of the Liver, 7th Edition, 2017, pg. 76-78;

[4] DRAKE R., VOGL A. W., MITCHELL A.W. M., Gray's Anatomy of the Human Body, 3th Edition, 2014, pg 121-125;

[5] Y. ISHAY, Y. ILAN, Hepatitis A and Other Viral Infections. Liver Immunology. 2020 Nov 4:227-53. doi: 10.1007/978-3-030-51709-0 15. PMCID: PMC7978675;

[6] R.M. PINTÓ, F.J. PÉREZ-RODRÍGUEZ, M.I. COSTAFREDA, G. CHAVARRIA-MIRÓ, S. GUIX, E. RIBES, A. BOSCH, Pathogenicity and virulence of hepatitis A virus. Virulence. 2021 Dec;12(1):1174-1185. doi: 10.1080/21505594.2021.1910442. PMID: 33843464; PMCID: PMC8043188;

[7] L. ZHANG, Hepatitis A vaccination. Hum Vaccin Immunother. 2020 Jul 2;16(7):1565-1573. doi: 10.1080/21645515.2020.1769389. Epub 2020 Jul 10. PMID: 32649265; PMCID: PMC7482852;

[8] C. HERZOG, K. VAN HERCK, P. VAN DAMME, Hepatitis A vaccination and its immunological and epidemiological long-term effects - a review of the evidence. Hum Vaccin Immunother. 2021;17(5):1496-1519. doi:10.1080/21645515.2020.1819742;

[9] T. KANDA, R. SASAKI, R. MASUZAKI, H. TAKAHASHI, T. MIZUTANI, N. MATSUMOTO, K. NIREI, M. MORIYAMA, Co-Occurrence of Hepatitis A Infection and Chronic Liver Disease. Int J Mol Sci. 2020 Sep 2;21(17):6384. doi: 10.3390/ijms21176384. PMID: 32887515; PMCID: PMC7504211;

[10] M.H. NGUYEN, G. WONG, E. GANE, J.H. KAO, G. DUSHEIKO, Hepatitis B Virus: Advances in Prevention, Diagnosis, and Therapy. Clin Microbiol Rev. 2020 Feb 26;33(2):e00046-19. doi: 10.1128/CMR.00046-19. PMID: 32102898; PMCID: PMC7048015;

[11] A. DAWOOD, S. ABDUL BASIT, M. JAYARAJ, R.G. GISH, Drugs in Development for Hepatitis B. Drugs. 2017 Aug;77(12):1263-1280. doi: 10.1007/s40265-017-0769-2. PMID: 28660478; PMCID: PMC5529495;

[12] E. SPYROU, C.I. SMITH, M.G. GHANY, Hepatitis B: Current Status of Therapy and Future Therapies. Gastroenterol Clin North Am. 2020 Jun;49(2):215-238. doi: 10.1016/j.gtc.2020.01.003. Epub 2020 Mar 29. PMID: 32389360; PMCID: PMC7444867;

[13] Y.F. SHIH, C.J. LIU, Hepatitis C Virus and Hepatitis B Virus Co-Infection. Viruses. 2020 Jul 
10;12(7):741. doi: 10.3390/v12070741. PMID: 32664198; PMCID: PMC7412310;

[14] M.A. MARTINEZ, S. FRANCO, Therapy Implications of Hepatitis C Virus Genetic Diversity. Viruses. 2020 Dec 29;13(1):41. doi: 10.3390/v13010041. PMID: 33383891; PMCID: PMC7824680;

[15] J.D. DUNCAN, R.A. URBANOWICZ, A.W. TARR, J.K. BALL, Hepatitis C Virus Vaccine: Challenges and Prospects. Vaccines (Basel). 2020 Feb 17;8(1):90. doi: 10.3390/vaccines8010090. PMID: 32079254; PMCID: PMC7157504;

[16] V.A. MOROZOV, S. LAGAYE, Hepatitis C virus: Morphogenesis, infection and therapy. World J Hepatol. 2018 Feb 27;10(2):186-212. doi: 10.4254/wjh.v10.i2.186. PMID: 29527256; PMCID: PMC5838439;

[17] D.B. NGUYEN, D. BIXLER, P.R. PATEL, Transmission of hepatitis C virus in the dialysis setting and strategies for its prevention. Semin Dial. 2019 Mar;32(2):127-134. doi: 10.1111/sdi.12761. Epub 2018 Dec 19. PMID: 30569604; PMCID: PMC6411055;

[18] A. RAJABI, M. AGHASI \& A. ROODBARI (2021). Biological monitoring of heavy metals in blood of Kerman residents, southeast of Iran. Technium BioChemMed, 2(2), 41-50.

[19] M. JIMÉNEZ-PÉREZ, R. GONZÁLEZ-GRANDE, P. ESPAÑA CONTRERAS, I. PINAZO MARTÍNEZ, J. DE LA CRUZ LOMBARDO, R. OLMEDO MARTÍN, Treatment of chronic hepatitis $\mathrm{C}$ with direct-acting antivirals: The role of resistance. World J Gastroenterol. 2016 Aug 7;22(29):6573 81. doi: 10.3748/wjg.v22.i29.6573. PMID: 27547001; PMCID: PMC4970473;

[20] A.A. PATEL, A. BUI, E. PROHL, D. BHATTACHARYA, S. WANG, A.D. BRANCH, P.V. PERUMALSWAMI, Innovations in Hepatitis C Screening and Treatment. Hepatol Commun. 2020 Dec 7;5(3):371-386. doi: 10.1002/hep4.1646. PMID: 33681673; PMCID: PMC7917266; 